\title{
Increased efficiency in biomimetic Lewis acid-base pair catalyzed monoacylation of diols by acyl phosphate monoesters
}

\author{
Yuyang $\mathrm{Li}^{\mathrm{a}}$ and Ronald Kluger ${ }^{\mathrm{a} \star}$ \\ ${ }^{a}$ Davenport Laboratories, Department of Chemistry, University of Toronto, 80 St. George Street, Toronto, \\ ON M5S 3H6, Canada \\ *.kluger@utoronto.ca
}

\section{Abstract}

Acyl phosphate monoesters are biomimetic acylation reagents that require coordination to metal ions to react with cis-diol substrates in water. With lanthanide catalysts, outcomes are compromised by (1) the competitive lanthanide-promoted hydrolysis of the acyl phosphate reagents as well as by (2) the high affinity of lanthanum ions for the phosphate monoester by-product. Based on analysis of the mechanism of the process, optimizing reaction conditions can selectively inhibit the lanthanum-promoted hydrolysis of acyl phosphate monoesters. Furthermore, using zinc salts and lead salts in place of lanthanides enhances the reactivity of the reactants and causes less complexation of the metal ion with the by-products.

\section{OPEN ACCESS}

Citation: Li Y and Kluger R. 2017. Increased efficiency in biomimetic Lewis acid-base pair catalyzed monoacylation of diols by acyl phosphate monoesters. FACETS 2: 682-689. doi: 0.1 I 39/facets-20 I 7-0047

Editor: Maxim V. Berezovski

Received: April 26, 2017

Accepted: July 6, 2017

Published: September 5, 2017

Copyright: (C) $2017 \mathrm{Li}$ and Kluger. This work is licensed under a Creative Commons Attribution 4.0 International License (CC BY 4.0), which permits unrestricted use, distribution, and reproduction in any medium, provided the original author(s) and source are credited.

Published by: Canadian Science Publishing

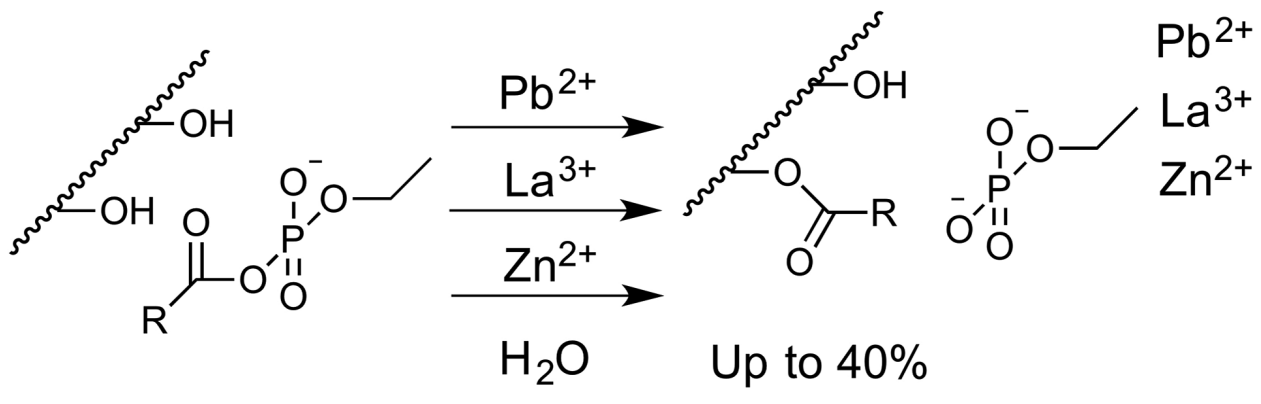

Key words: biomimetic acylation, Lewis acid catalysis, acyl phosphate esters, zinc and lead ion catalysts, cis-diol substrates, monoesters

\section{Introduction}

The utility of acyl phosphates esters as biomimetic reagents for selective monoacylation of diols is a subject of continuing interest with important applications, including kinome interrogation (Patricelli et al. 2007; Nordin et al. 2015) and aminoacylation of the 3 '-terminus of tRNA (Tzvetkova and Kluger 2007; Duffy and Dougherty 2010). We previously reported that lanthanide ions can serve as the chelated core to bind and activate the reacting species toward acyl transfer (Cameron et al. 2004; Tzvetkova and Kluger 2007; Her and Kluger 2011). Bidentate coordination of a cis-1,2-diol to a Lewis acid facilitates ionization of one of the coordinated diols while enhancing the activity of the bidentate-coordinate acyl phosphate (Scheme 1). The bis-bidentate array of 


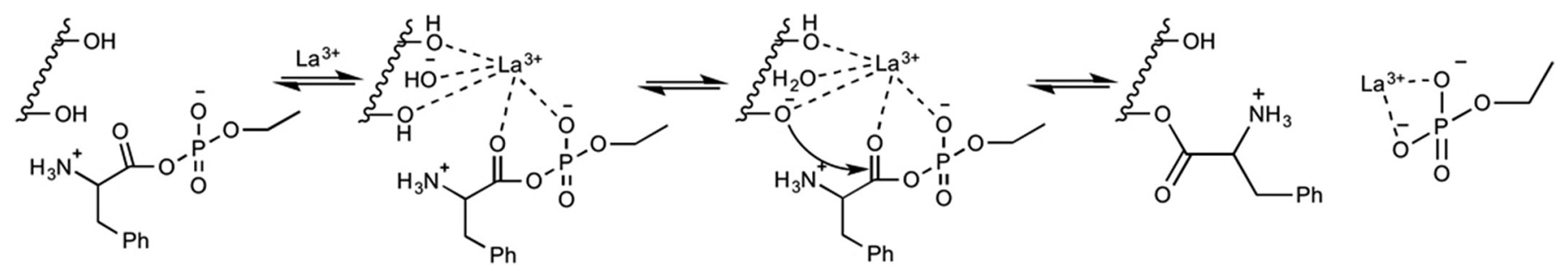

Scheme 1. Lanthanum-promoted monoacylation of the diol in a nucleotide via a chelate formation.

coordinated Lewis base (diol) and Lewis acid (acyl phosphate) promotes the ultimate combination that produces the acylated diol along with a phosphate monoester as a by-product. The arrangement can utilize the potential energy made available by the separation of the Lewis acid-base pair. Utilizing acyl phosphate monoesters as metal-coordinated acyl donors at the same Lewis acid where the ionized diol is coordinated promotes the reaction without involvement of the solvent. With a similar approach, regioselective monoacylation of carbohydrates in water can be based on the geometry of adjacent hydroxyl groups (Gray and Kluger 2007; Dhiman and Kluger 2010). However, although the system is set up for efficient acylation, the formation of the correct combination of ligands in the chelate for reaction is subject to competing homologous formation of the chelates as well as interference due to coordination to the lanthanide of the phosphate monoester product (see below).

An important potential use of the approach is for biomimetic aminoacylation of tRNA for the ribosomal introduction of unnatural amino acids into new proteins, which requires an aminoacylated reagent whose reactive functionality parallels that of the biological agents, aminoacyl adenylates (Schimmel 1987). However, complications arise from the competing effectiveness of the lanthanum ion in the hydrolysis of phosphate derivatives that are the acylation reagents (Hendry and Sargeson 1989; Kluger et al. 1997). Furthermore, transfer of the acyl group, whether to the hydroxyl or to water, releases ethyl phosphate, forming an insoluble complex with the metal ions that prevents further catalysis (Tzvetkova 2008).

In the present study, we assessed the effects of other metal ions for reactions in water that are reported to be effective in nonaqueous systems (Hikawa et al. 2014). We also assessed aqueous reaction conditions with added solvents and at lower reaction temperatures. In some cases, these provided significant improvements for the overall process.

\section{Experimental}

\section{Preparation of N-deprotected phenylalanyl ethyl phosphate}

BOC-PheEtP was formed in the DCC-mediated coupling of BOC-Phe and ethyl phosphate. PheEtP was produced by removal of the BOC protecting group using a minimal amount of trifluoroacetic acid. The N-deprotected product was precipitated from ice-cold dry acetone as a white powder (Kluger et al. 1996). ESI-MS [M- $\left.\mathrm{H}^{+}\right]$calculated $m / z=272.0693$ and found $m / z=272.0711$.

\section{Analysis of the extent of PheEtP hydrolysis}

A mixture of PheEtP $(50 \mathrm{mmol} / \mathrm{L})$ and lanthanum triflate $(50 \mathrm{mmol} / \mathrm{L})$ was incubated in an aqueous MES buffer (200 mmol/L, pH 6). We assessed the effects of conducting the reaction in a range of DMSO/buffer mixtures, as follows: $0 / 1,2 / 3,3 / 2,9 / 1,19 / 1$, and 39/1 $(v / v)$. The effect of temperature on product yield was studied by conducting reactions at 20 and $4{ }^{\circ} \mathrm{C}$. Samples were analyzed after periodic additions of $20 \%$ volume of saturated EDTA solution until no further changes were observed. Hydrolysis of PheEtP was then tracked by the integrated peak area from UV-HPLC at $260 \mathrm{~nm}$. 


\section{Monoaminoacylation of ethylene glycol}

A mixture of ethylene glycol $(60 \mathrm{mmol} / \mathrm{L})$, PheEtP $(50 \mathrm{mmol} / \mathrm{L})$, and metal salt $\left(\mathrm{La}(\mathrm{OTf})_{3}=\right.$ $\left.\mathrm{Zn}\left(\mathrm{NO}_{3}\right)_{2}=\mathrm{Pb}\left(\mathrm{NO}_{3}\right)_{2}=50 \mathrm{mmol} / \mathrm{L}\right)$ was stirred in an MES buffer $(200 \mathrm{mmol} / \mathrm{L}, \mathrm{pH} 6)$ at $4{ }^{\circ} \mathrm{C}$ for 24-72 h. Samples were analyzed after periodic additions of $20 \%$ volume of saturated EDTA solution until no further changes were observed. Products were isolated by reversed phase HPLC and lyophilized. A colorless solid was collected. The yield was estimated from the integration of the peak areas from UV-HPLC at $260 \mathrm{~nm}$. ESI-MS $\left[\mathrm{M}+\mathrm{H}^{+}\right]$calculated $\mathrm{m} / z=210.11$ and found $\mathrm{m} / z=210.1$.

\section{Monobenzoylation of ethylene glycol}

A mixture of ethylene glycol $(60 \mathrm{mmol} / \mathrm{L}), \mathrm{BMP}(50 \mathrm{mmol} / \mathrm{L})$, and metal salt $\left(\mathrm{La}(\mathrm{OTf})_{3}=\right.$ $\left.\mathrm{Zn}\left(\mathrm{NO}_{3}\right)_{2}=\mathrm{Pb}\left(\mathrm{NO}_{3}\right)_{2}=50 \mathrm{mmol} / \mathrm{L}\right)$ was stirred in an EPPS buffer $(200 \mathrm{mmol} / \mathrm{L} \mathrm{pH} 8)$ at $25^{\circ} \mathrm{C}$ for 24-72 h (Cameron et al. 2004). Samples were analyzed after periodic additions of $20 \%$ volume of saturated EDTA solution until no further changes were observed. Products were isolated by reversed phase HPLC and lyophilized. A colorless solid was collected. The yield was estimated from the integration of the peak areas from UV-HPLC at $230 \mathrm{~nm}$.

\section{Preparation of 5'-phospho-2'-deoxyribocytidylylriboadenosine}

dCA was produced following published methods (Noren et al. 1989). ESI-MS [M+ $\left.\mathrm{H}^{+}\right]$calculated $m / z=637.1167$ and found $m / z=637.1173$.

\section{Aminoacylation of nucleotides}

AMP $(50 \mathrm{mmol} / \mathrm{L})$ and dCA $(1 \mathrm{mmol} / \mathrm{L})$ were reacted with PheEtP $(50 \mathrm{mmol} / \mathrm{L})$ and metal salts $(50 \mathrm{mmol} / \mathrm{L})$ as described for the reaction with ethylene glycol. ESI-MS $\left[\mathrm{M}+\mathrm{H}^{+}\right]$for AMP-Phe: calculated $\mathrm{m} / z=493.1242$ and found $\mathrm{m} / z=493.1197$; for dCA-Phe: calculated $\mathrm{m} / z=784.2000$ and found $m / z=784.2000$.

\section{Results and discussion}

An aminoacyl phosphate monoester, PheEtP, was used as the acylation agent for the evaluation of catalysts in this study. This reagent is a good model for the general approach to the formation of amino acids esters at the $3^{\prime}$-terminal of tRNA. We found that conducting reactions in mixtures of DMSO and water reduced the rate of hydrolysis of PheEtP in the presence of lanthanum ion compared with the reaction in water alone. In a 95/5 DMSO/aqueous buffer $(v / v)$, the $t_{1 / 2}$ of PheEtP was about $30 \mathrm{~min}$, whereas the $t_{1 / 2}$ in water was $<2 \mathrm{~min}$. Nonetheless, the yield of acylated products did not increase with the reduced hydrolysis rate of the reagent. Surprisingly, the yield of ester derivatives that were formed at the $2^{\prime}$ - or $3^{\prime}$-ribosyl positions of AMP with PheEtP remained near $30 \%$ after $24 \mathrm{~h}$, regardless of the amount of DMSO in the aqueous reaction mixture. This suggests that the reactants are locally solvated exclusively by water. The polarity of the reaction partners and their ability to form hydrogen bonds is consistent with this outcome. It is likely that the ionization of water coordinated to lanthanum ions is essential for the activation of PheEtP, whereas the reactivity of the hydroxyl group of diol toward the carboxyl carbon is increased as it is deprotonated by the lanthanum-coordinated hydroxyl (Kluger and Cameron 2002). Lowering the relative amount of water reduces the extent of ionization of the hydroxyl of the diol moiety, reducing the reactivity in the coordinated ligands.

The problematic competing hydrolysis of PheEtP occurs by the addition of non-chelated water. This will have a higher enthalpic barrier if it avoids the coordination sphere of the metal ion. Lowering the reaction temperature should reduce the rate of the reaction with water to a greater extent than it will reduce the desired acylation from reaction with the bis-bidentate coordinated chelate. As expected, at $4{ }^{\circ} \mathrm{C}$, hydrolysis occurred at a significantly slower rate than at room temperature. At the lower 
temperature, $15 \%$ of the initial PheEtP remained after $2.5 \mathrm{~h}$, corresponding to a $t_{1 / 2}$ of $1 \mathrm{~h}$ under these conditions. Applying the same conditions to the lanthanum ion-promoted aminoacylation of AMP increased the yield from around $30 \%$ to $40 \%$. For aminoacylation of dCA, a dinucleotide used by Robertson et al. (1989) in the chemical aminoacylation of tRNA, the combined yields of $2^{\prime}$ and $3^{\prime}$ esters doubled from $6.8 \%$ to $15 \%$ after $12 \mathrm{~h}$ of reaction. With PheEtP at its saturating concentration, the reaction produced the desired esters at a $40 \%$ yield after $12 \mathrm{~h}$ at $4{ }^{\circ} \mathrm{C}$ (Table 1 ). This establishes the importance of using lower reaction temperatures, consistent with the expected effect of selectively slowing the competing hydrolysis.

With this useful outcome, we optimized conditions for acylation with metal salts and determined the extent of the competing hydrolysis of PheEtP. The $t_{1 / 2}$ for catalyzed hydrolysis of PheEtP at $4{ }^{\circ} \mathrm{C}$ in the presence of zinc or lead salts were 60 and $5 \mathrm{~min}$, respectively. The reaction in the presence of cupric salts is not effective, as it selectively promotes the hydrolysis of PheEtP. Based on these observations, we anticipated that $\mathrm{Zn}^{2+}$ and $\mathrm{Pb}^{2+}$ should catalyze aminoacylation of diols at or near $0{ }^{\circ} \mathrm{C}$. We tested this approach by following the aminoacylation of ethylene glycol by PheEtP at $4{ }^{\circ} \mathrm{C}$ (Table 2).

Table 1. Yields for aminoacylation of AMP and dCA by PheEtP in the presence of Lewis acids.

\begin{tabular}{lccc} 
Salt & Nucleotide & Temperature $\left({ }^{\circ} \mathrm{C}\right)$ & Combined yield \\
$\mathrm{La}(\mathrm{OTf})_{3}$ & AMP & 25 & $29 \%$ \\
& & 4 & $36 \%$ \\
& $\mathrm{dCA}$ & 25 & $6.8 \%$ \\
$\mathrm{Zn}\left(\mathrm{NO}_{3}\right)_{2}$ & 4 & $15 \%(40 \%)$ \\
& AMP & 4 & $31 \%$ \\
$\mathrm{~Pb}\left(\mathrm{NO}_{3}\right)_{2}$ & dCA & 4 & $19 \%$ \\
& AMP & 4 & $31 \%$ \\
\hline
\end{tabular}

Note: AMP, adenosine monophosphate; dCA, 5'-phospho-2'-deoxyribocytidylylriboadenosine; PheEtP, phenylalanyl ethyl phosphate.

Table 2. Summary of metal-mediated monoacylation reaction of ethylene glycol with PheEtP and BMP.

$\begin{array}{lccc}\text { Acyl phosphate } & \text { Salt } & \text { [Salt] }(\mathrm{mmol} / \mathrm{L}) & \text { Yield } \\ \text { PheEtP } & 50 & 64 \% \\ & \mathrm{La}(\mathrm{OTf})_{3} & 50 & 23 \% \\ & \mathrm{Zn}\left(\mathrm{NO}_{3}\right)_{2} & 150 & 24 \% \\ & & 50 & 22 \% \\ & \mathrm{ZnSO}_{4} & 50 & 67 \% \\ & \mathrm{~Pb}\left(\mathrm{NO}_{3}\right)_{2} & 150 & 63 \% \\ & & 50 & 3.5 \% \\ \mathrm{BMP} & \mathrm{La}(\mathrm{OTf})_{3} & 50 & 3.6 \% \\ & \mathrm{Zn}\left(\mathrm{NO}_{3}\right)_{2} & 50 & 7.5 \% \\ & \mathrm{~Pb}\left(\mathrm{NO}_{3}\right)_{2} & \end{array}$

Note: PheEtP, phenylalanyl ethyl phosphate; BMP, benzoyl methyl phosphate. 
In the presence of $\mathrm{Zn}^{2+}$ or $\mathrm{Pb}^{2+}$, the reactions of ethylene glycol and PheEtP produced a mixture of the ester and the hydrolysis product, phenylalanine, within $1 \mathrm{~min}$ after addition to the solution. The $\mathrm{Pb}^{2+}$-catalyzed reactions occurred with a half-life for PheEtP of $<1 \mathrm{~min}$. The yields are comparable with those from reactions conducted in the presence of $\mathrm{La}^{3+}$. The $\mathrm{Zn}^{2+}$-catalyzed reactions were slower and their overall yields were lower than with $\mathrm{Pb}^{2+}$ and $\mathrm{La}^{3+}$.

We also investigated the reactions of ethylene glycol with BMP (Scheme 2). The $t_{1 / 2}$ for hydrolysis of BMP in the presence of $\mathrm{Zn}^{2+}$ and $\mathrm{Pb}^{2+}$ were 24 and $1 \mathrm{~h}$, respectively. The $\mathrm{Pb}^{2+}$-catalyzed monobenzoylation reactions of ethylene glycol gave the best yield of the monoester after $3 \mathrm{~h}$, whereas reactions with $\mathrm{Zn}^{2+}$ required about $12 \mathrm{~h}$ to reach their maximum yield.

We also evaluated the effectiveness of $\mathrm{Zn}^{2+}$ and $\mathrm{Pb}^{2+}$ as catalysts for reactions of PheEtP with a mononucleotide and a dinucleotide. Both AMP and dCA were separately incubated with PheEtP in the presence of the metal ions at $4{ }^{\circ} \mathrm{C}$, followed by analysis that used reverse-phase HPLC to separate the reactants and products. The reactions gave $2^{\prime}$ and $3^{\prime}$ nucleotidyl esters in each case. In reactions with dCA, the yield was $19 \%$ with $\mathrm{Zn}^{2+}$ and $13 \%$ with $\mathrm{Pb}^{2+}$ (Figures 1 and 2 ). These did not improve on the previously reported $15 \%$ yield with $\mathrm{La}^{3+}$. We also monitored the reactions for an additional $72 \mathrm{~h}$ during which the distribution of products remained unchanged, establishing that the esters are stable under these conditions and that the reactions are self-limiting.

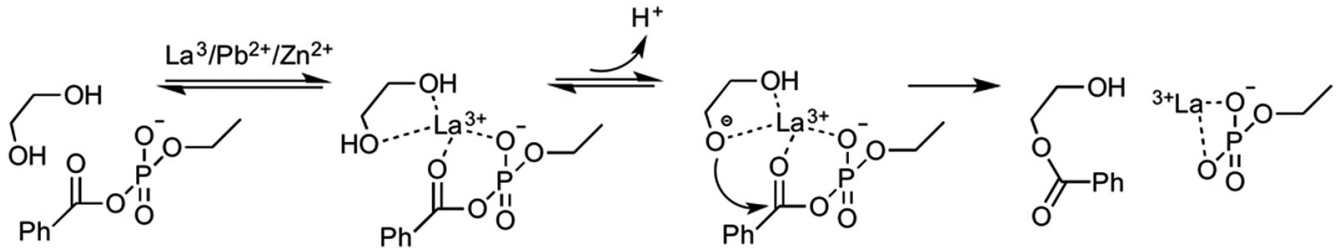

Scheme 2. Lanthanum/lead/zinc-mediated monobenzoylation of ethylene glycol.

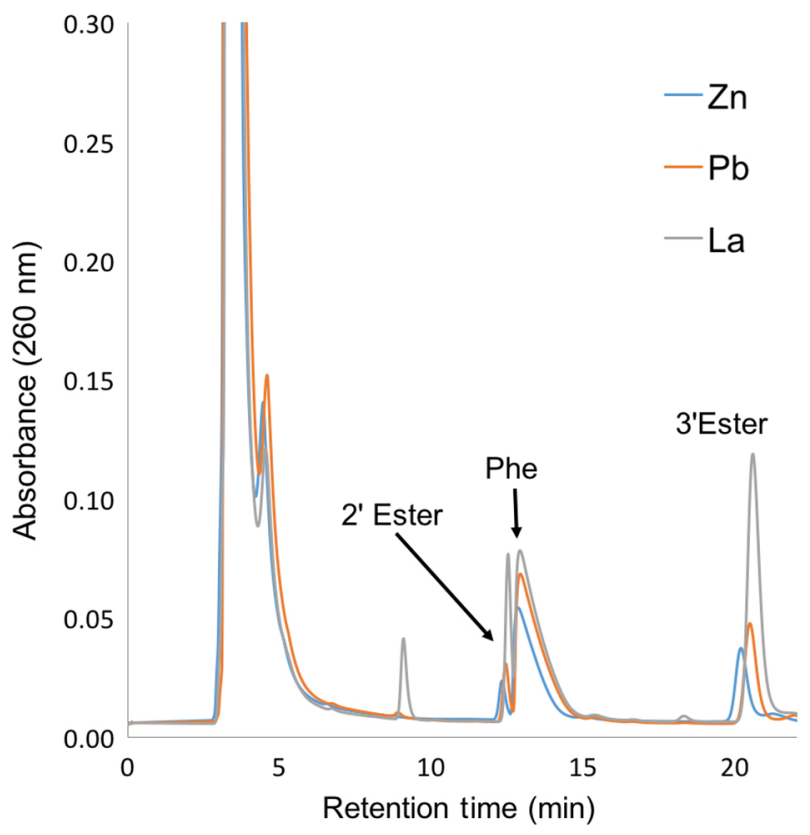

Fig. 1. Amionacylation reaction with adenosine monophosphate (AMP) and phenylalanyl ethyl phosphate (PheEtP) catalyzed by $\mathrm{La}^{3+}, \mathrm{Pb}^{2+}$, and $\mathrm{Zn}^{2+}$. 


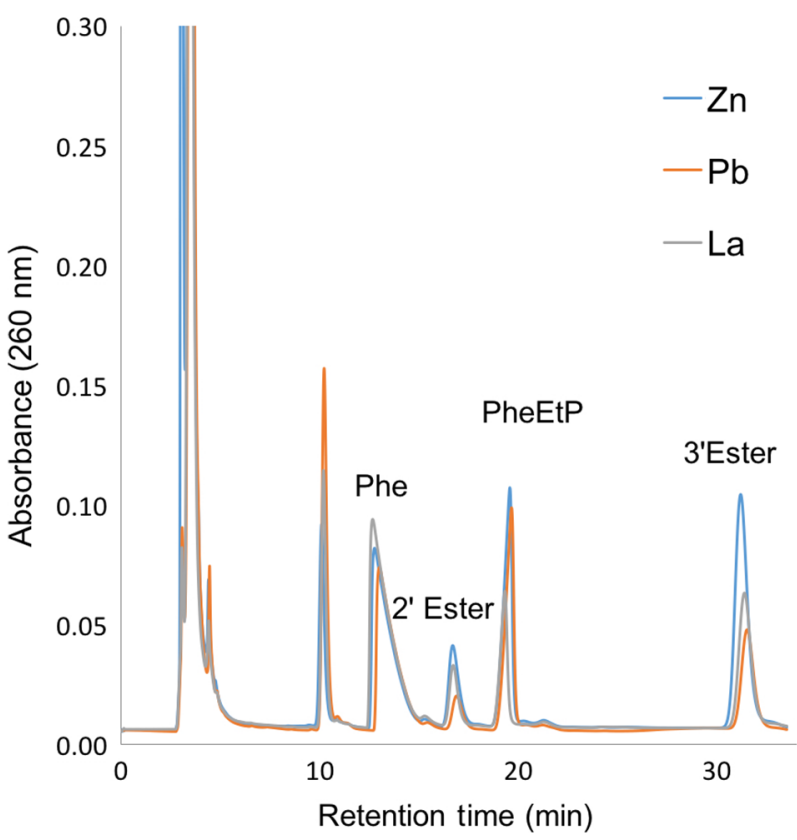

Fig. 2. Aminoacylation reaction with 5'-phospho-2'-deoxyribocytidylylriboadenosine (dCA) and phenylalanyl ethyl phosphate (PheEtP) catalyzed by $\mathrm{La}^{3+}$, $\mathrm{Pb}^{2+}$, and $\mathrm{Zn}^{2+}$

Reports of previous studies indicate that $\mathrm{Pb}^{2+}$ is among the most effective catalysts for site-specific cleavage of RNA (Huff et al. 1964; Brown et al. 1983). Lead hydroxide is an active catalyst for RNA hydrolysis (Brown et al. 1983) and for transesterification of a phosphate diester (Morrow et al. 1992). In reactions at $\mathrm{pH}$ around 7 , where $\mathrm{Pb}^{2+}$-promoted aminoacylation occurs, lead hydroxides are present (Perera et al. 2001). Lead hydroxides could assist in deprotonation of the incoming hydroxyl, further promoting acylation.

As noted earlier, a major problem with $\mathrm{La}^{3+}$-promoted acylation is the high affinity of $\mathrm{La}^{3+}$ to phosphate monoesters. The resulting precipitate removes the $\mathrm{La}^{3+}$ catalyst from solution. As a result, $\mathrm{La}^{3+}$-promoted acylation reactions require a large excess of the added metal ion (Gray and Kluger 2007). Reactions with $\mathrm{Zn}^{2+}$ and $\mathrm{Pb}^{2+}$ reduce the formation of insoluble metal phosphates, providing alternative catalysts for acylation reactions via acyl phosphates that can operate at lower concentrations.

\section{Conclusions}

We have demonstrated that lowering the reaction temperature improves lanthanide-ion-promoted aminoacylation reactions by selectively slowing the competing hydrolysis of activated amino acyl phosphates. Catalysis by $\mathrm{Zn}^{2+}$ and $\mathrm{Pb}^{2+}$ is effective, producing the desired aminoacylation products under conditions where the acyl phosphate reagent has improved stability.

\section{List of abbreviations}

$\begin{array}{ll}\text { AMP } & \text { adenosine } 5^{\prime} \text {-monophosphate } \\ \text { BMP } & \text { benzoyl methyl phosphate } \\ \text { BOC } & \text { tertbutyl oxycarbonyl } \\ \text { dCA } & 5^{\prime} \text {-phospho-2'-deoxyribocytidylylriboadenosine } \\ \text { DCC } & \mathrm{N}, \mathrm{N}^{\prime} \text {-dicyclohexylcarbodiimide } \\ \text { DMSO } & \text { dimethyl sulfoxide } \\ \text { EDTA } & \text { ethylenediamine tetraacetic acid }\end{array}$




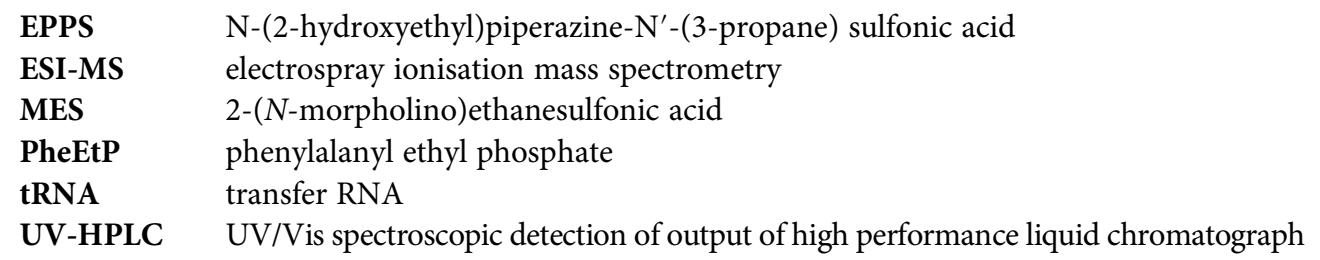

\section{Acknowledgements}

We thank NSERC Canada for support through a Discovery Grant.

\section{Author contributions}

YL and RK conceived and designed the study. YL performed the experiments/collected the data. YL and RK analyzed and interpreted the data. RK contributed resources. YL and RK drafted or revised the manuscript.

\section{Competing interests}

RK is currently serving as a Subject Editor for FACETS, but was not involved in review or editorial decisions regarding this manuscript.

\section{Data accessibility statement}

All relevant data are within the paper.

\section{References}

Brown RS, Hingerty BE, Dewan JC, and Klug A. 1983. $\mathrm{Pb}$ (II)-catalysed cleavage of the sugarphosphate backbone of yeast tRNA ${ }^{\text {Phe }}$-implications for lead toxicity and self-splicing RNA. Nature, 303(5917): 543-546. PMID: 6343887 DOI: 10.1038/303543a0

Cameron LL, Wang SC, and Kluger R. 2004. Biomimetic monoacylation of diols in water. Lanthanidepromoted reactions of methyl benzoyl phosphate. Journal of the American Chemical Society, 126(34): 10721-10726. PMID: 15327331 DOI: 10.1021/ja0495381

Dhiman RS, and Kluger R. 2010. Magnesium ion enhances lanthanum-promoted monobenzoylation of a monosaccharide in water. Organic \& Biomolecular Chemistry, 8(9): 2006-2008. PMID: 20401373 DOI: $10.1039 / \mathrm{b} 926851 \mathrm{k}$

Duffy NH, and Dougherty DA. 2010. Preparation of translationally competent tRNA by direct chemical acylation. Organic Letters, 12(17): 3776-3779. PMID: 20687605 DOI: 10.1021/ol101408f

Gray IJ, and Kluger R. 2007. Chelation-controlled regioselectivity in the lanthanum-promoted monobenzoylation of monosaccharides in water. Carbohydrate Research, 342(14): 1998-2002. PMID: 17572400 DOI: $10.1016 /$ j.carres.2007.05.024

Hendry P, and Sargeson AM. 1989. Metal ion promoted phosphate ester hydrolysis. Intramolecular attack of coordinated hydroxide ion. Journal of the American Chemical Society, 111(7): 2521-2527. DOI: $10.1021 / \mathrm{ja} 00189 \mathrm{a} 025$

Her S, and Kluger R. 2011. Biomimetic protecting-group-free 2', 3'-selective aminoacylation of nucleosides and nucleotides. Organic \& Biomolecular Chemistry, 9(3): 676-678. PMID: 21132175 DOI: $10.1039 / \mathrm{COOB} 00795 \mathrm{~A}$ 
Hikawa H, Hamada M, Yokoyama Y, and Azumaya I. 2014. Benzoyl methyl phosphate as an efficient reagent for the selective monobenzoylation of N-Bz-FTY720. RSC Advances, 4(44): 23131-23136. DOI: $10.1039 /$ C4RA02401J

Huff JW, Sastry KS, Gordon MP, and Wacker WEC. 1964. The action of metal ions on tobacco mosaic virus ribonucleic acid. Biochemistry, 3(4): 501-506. PMID: 14188164 DOI: 10.1021/ bi00892a006

Kluger R, and Cameron LL. 2002. Activation of acyl phosphate monoesters by lanthanide ions: enhanced reactivity of benzoyl methyl phosphate. Journal of the American Chemical Society, 124(13): 3303-3308. PMID: 11916414 DOI: 10.1021/ja016600v

Kluger R, Li X, and Loo RW. 1996. 1996 Bader Award Lecture Aminoacyl ethyl phosphates. Biomimetically activated amino acids. Canadian Journal of Chemistry, 74(12): 2395-2400. DOI: $10.1139 / \mathrm{v} 96-268$

Kluger R, Loo RW, and Mazza V. 1997. Biomimetically activated amino acids. Catalysis in the hydrolysis of alanyl ethyl phosphate. Journal of the American Chemical Society, 119(50): 12089-12094. DOI: 10.1021/ja972406q

Morrow JR, Buttrey LA, and Berback KA. 1992. Transesterification of a phosphate diester by divalent and trivalent metal ions. Inorganic Chemistry, 31(1): 16-20. DOI: 10.1021/ic00027a005

Nordin BE, Liu Y, Aban A, Brown HE, Wu J, Hainley AK, et al. 2015. ATP acyl phosphate reactivity reveals native conformations of Hsp90 paralogs and inhibitor target engagement. Biochemistry, 54(19): 3024-3036. PMID: 25905789 DOI: 10.1021/acs.biochem.5b00148

Noren CJ, Anthony-Cahill SJ, Griffith MC, and Schultz PG. 1989. A general method for site-specific incorporation of unnatural amino acids into proteins. Science, 244(4901): 182-188. PMID: 2649980 DOI: $10.1126 /$ science. 2649980

Patricelli MP, Szardenings AK, Liyanage M, Nomanbhoy TK, Wu M, Weissig H, et al. 2007. Functional interrogation of the kinome using nucleotide acyl phosphates. Biochemistry, 46(2): 350-358. PMID: 17209545 DOI: 10.1021/bi062142x

Perera WN, Hefter G, and Sipos PM. 2001. An investigation of the lead (II)-hydroxide system. Inorganic Chemistry, 40(16): 3974-3978. PMID: 11466056 DOI: 10.1021/ic001415o

Robertson SA, Noren CJ, Anthony-Cahill SJ, Griffith MC, and Schultz PG. 1989. The use of 5 '-phospho-2 deoxyribocytidylylriboadenosine as a facile route to chemical aminoacylation of tRNA. Nucleic Acids Research, 17(23): 9649-9660. PMID: 2602139 DOI: 10.1093/nar/17.23.9649

Schimmel P. 1987. Aminoacyl tRNA synthetases: general scheme of structure-function relationships in the polypeptides and recognition of transfer RNAs. Annual Review of Biochemistry, 56: 125-158. PMID: 3304131 DOI: 10.1146/annurev.bi.56.070187.001013

Tzvetkova S. 2008. Biomimetic aminoacylation. Ph.D. thesis, University of Toronto, Toronto, Ontario. 104 p.

Tzvetkova S, and Kluger R. 2007. Biomimetic aminoacylation of ribonucleotides and RNA with aminoacyl phosphate esters and lanthanum salts. Journal of the American Chemical Society, 129(51): 15848-15854. PMID: 18052163 DOI: 10.1021/ja0739761 